\title{
Defining Objects for in-Depth Examination of Energy in Infrastructure Budget Organizations and Institutions at the Municipal Level
}

\author{
Evgenia U. Sizganova**, \\ Roman A. Petukhov and Dmitry V. Antonenkov \\ ${ }^{a}$ Siberian Federal University \\ 79 Svobodny, Krasnoyarsk, 660041, Russia \\ ${ }^{b}$ Northeast Federal University M.K. Ammosov's name \\ 16 Kravchenko, Nerungry, 678960, Russia
}

Received 18.11.2014, received in revised form 10.01.2015, accepted 06.02.2015

Considered the way of determining the objects with the irrational power consumption for thorough energy survey on the basis of the index of viability objects on power consumption. Using the method allows to make additional research of objects and to draw conclusions about the dynamics of their development as a whole. The method is implemented in the package MathCAD and presented on the example of the infrastructure of educational institutions Neryungri District.

Keywords: irrational power consumption, energy survey, the index of viability, rank analysis.

\section{Определение объектов}

\section{для углубленного энергообследования}

в инфраструктуре бюджетных организаций

и учреждений муниципального уровня

Е.Ю. Сизганова ${ }^{a}$, Р.А. Петухов ${ }^{\text {a }}$, Д.В. Антоненков ${ }^{\tilde{\sigma}}$ ${ }^{a}$ Сибирский федеральный университет Россия, 660041, Красноярск, пр. Свободньій, 79

${ }^{6}$ Северо-Восточный федеральный университет им. М.К. Аммосова Россия, 678960, Республика Саха (Якутия), Нерюнгри, ул. Кравченко, 16

Рассмотрен способ определения объектов с нерачиональным электропотреблением для углубленного энергетического обследования на основе индекса жизнеспособности объектов

(C) Siberian Federal University. All rights reserved

* Corresponding author E-mail address: seu_eset@mail.ru 
по электропотреблению. Использование способа позволяет произвести дополнительное исследование объектов и сделать выводы относительно динамики их развития в целом. Способ реализован в пакете МатhCAD и представлен на примере инфраструктуры образовательных учреждений Нерюнгринского района.

Ключевые слова: нерачиональное электропотребление, энергетическое обследование, индекс жизнеспособности, ранговый анализ.

Одной из ключевых процедур управления инфраструктурой бюджетных организаций и учреждений муниципального уровня $[1,2]$ служит своевременное выявление первоочередных объектов для углубленного энергетического обследования. Процедура интервального оценивания позволяет установить и проранжировать объекты инфраструктуры, аномально потребляющие электроэнергию. Однако если таковых оказывается сравнительно много, то требуется дополнительное исследование для определения объектов, энергоаудит которых является первоочередной задачей для техноценоза в целом. Для этого осуществляется так называемое оценивание жизнеспособности объектов по электропотреблению [3-5]. Кроме того, данная процедура позволяет произвести дополнительное исследование объектов и сделать весьма плодотворные выводы относительно динамики их развития в целом (достаточно полно описано в [4]).

В ходе принятия решения по углубленному энергетическому обследованию объектов инфраструктуры бюджетных организаций и учреждений муниципального уровня в качестве ключевого макроиндикатора учитывается их индекс жизнеспособности [4]. Индексом жизнеспособности называется угол, образуемый с осью абсцисс линией тренда временного ряда относительного электропотребления объекта, которое определяется как отношение электропотребления каждого объекта к суммарному электропотреблению объектов инфраструктуры. Как показано в $[4,5]$, величина и знак индекса жизнеспособности позволяют сделать выводы относительно динамики развития (или стагнации) объекта, а также определить его номер в списке первоочередных объектов для углубленного энергетического обследования.

Для исследования использовали собранные за восемь лет данные по электропотреблению инфраструктуры образовательных учреждений (49 объектов), расположенных на территории Нерюнгринского района. Предварительно по результатам первичной обработки статистической информации по инфраструктуре образовательных учреждений [7] сформирован ряд рабочих файлов, которые будут применены в данной программе для осуществления операции определения первоочередных объектов для углубленного энергетического обследования.

\section{Подготовка данных}

Задаем начало отсчета ORIGIN $:=1$.

Считываем из файла информацию об исследуемой инфраструктуре в виде двумерной матрицы V, которая содержит эту информацию, причем каждая строка соответствует определенному временному интервалу (году), а каждый столбец - объекту.

$$
\mathrm{V}:=
$$


Транспонируем полученную матрицу $\mathrm{VT}:=\mathrm{V}^{\mathrm{T}}$ и определяем количество объектов [6]: $\mathrm{n}:=\operatorname{cols}(\mathrm{V})=49$.

\section{Расчет электропотребления инфраструктуры}

Необходимо определить отношение электропотребления каждого из объектов к общему суммарному электропотреблению инфраструктуры образовательных учреждений.

Листинг 1. Определение суммарного потребления электроэнергии инфраструктурой образовательных учреждений для каждого года.

$$
\operatorname{SUM}(M):=\mid \begin{aligned}
& \mathrm{S} \leftarrow 0 \\
& \mathrm{n} \leftarrow \operatorname{rows}(\mathrm{M}) \\
& \text { for } \mathrm{i} \in 1 \ldots \operatorname{cols}(\mathrm{M}) \\
& \mathrm{S}_{\mathrm{i}} \leftarrow \sum_{\mathrm{n}=1}^{\mathrm{n}} \mathrm{M}_{\mathrm{n}, \mathrm{i}} \\
& \mathrm{S}
\end{aligned}
$$

Листинг 2. Вычисление отношения электропотребления каждого из объектов к общему электропотреблению.

$$
\operatorname{DOL}(\mathrm{M}, \mathrm{SI}):=\mid \begin{aligned}
& \mathrm{S} \leftarrow 0 \\
& \mathrm{n} \leftarrow \operatorname{rows}(\mathrm{M}) \\
& \text { for } \mathrm{i} \in 1 . . \operatorname{cols}(\mathrm{M}) \\
& \mathrm{D}^{\langle\mathrm{i}\rangle} \leftarrow \frac{\mathrm{M}^{\langle\mathrm{i}\rangle}}{\mathrm{SI}_{\mathrm{i}}} \cdot 100 \\
& \mathrm{D}
\end{aligned}
$$

Результаты вычислений отображаются в процентах в матрице D, каждому столбцу которой соответствует объект, а строке - временной интервал.

$$
\mathrm{D}:=\mathrm{DOL}(\mathrm{VT}, \mathrm{SI})^{\mathrm{T}}
$$

$\mathrm{D}=$\begin{tabular}{|r|r|r|r|r|r|r|r|}
\hline & 1 & \multicolumn{1}{c|}{2} & \multicolumn{1}{c|}{3} & \multicolumn{1}{c|}{4} & \multicolumn{1}{c|}{5} & \multicolumn{1}{c|}{6} & 7 \\
\hline 1 & 3.999 & 4.739 & 2.546 & 3.564 & 4.762 & 4.858 & 6.578 \\
\hline 2 & 3.745 & 4.785 & 2.79 & 3.698 & 4.47 & 5.113 & 6.599 \\
\hline 3 & 3.514 & 4.362 & 2.599 & 3.783 & 3.936 & 4.603 & 6.616 \\
\hline 4 & 3.596 & 4.367 & 2.716 & 3.825 & 3.702 & 4.953 & 6.648 \\
\hline 5 & 4.234 & 4.344 & 2.222 & 4.161 & 4.044 & 3.616 & 6.934 \\
\hline 6 & 5.638 & 3.854 & 2.054 & 5.064 & 4.408 & 4.344 & 5.852 \\
\hline 7 & 5.356 & 4.184 & 1.698 & 6.628 & 4.453 & 4.291 & 5.449 \\
\hline 8 & 5.011 & 4.672 & 1.9 & 6.599 & 4.651 & 4.05 & $\ldots$ \\
\hline
\end{tabular}

Определяем количество временных интервалов (t) и визуализируем расчеты с помощью графика (рис. 1): 


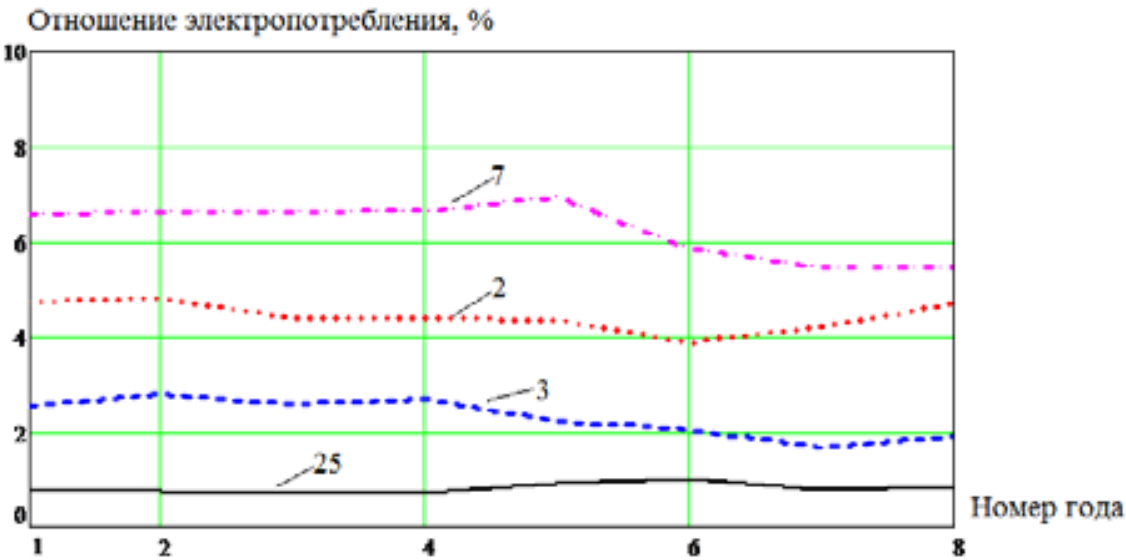

Рис. 1. Временные ряды относительного электропотребления образовательных учреждений Нерюнгринского района

На рис. 1 в качестве примера построены графики только для объектов с номерами 2, 3, 7 и 25, причем самый верхний график соответствует номеру 7 (средняя школа № 24), ниже идет 2 (средняя школа № 2), затем 3 (средняя школа № 3) и ниже всех 25 (д/с «Солнышко»).

\section{Расчет индекса жизнеспособности объектов по электропотреблению}

Рассчитываем тренд временных рядов относительного электропотребления для каждого из объектов инфраструктуры. Как было отмечено, угол наклона тренда к оси абсцисс отражает динамику изменения доли электропотребления каждого объекта в общем электропотреблении техноценоза и тем самым служит макроиндикатором жизнеспособности объекта по электропотреблению.

В качестве тренда используется аппроксимирующая прямая.

Листинг 3. Вычисление коэффициентов регрессии методом наименьиих квадратов.

$$
\begin{aligned}
& \mathrm{X}_{\mathrm{i}}:=\mathrm{i}
\end{aligned}
$$

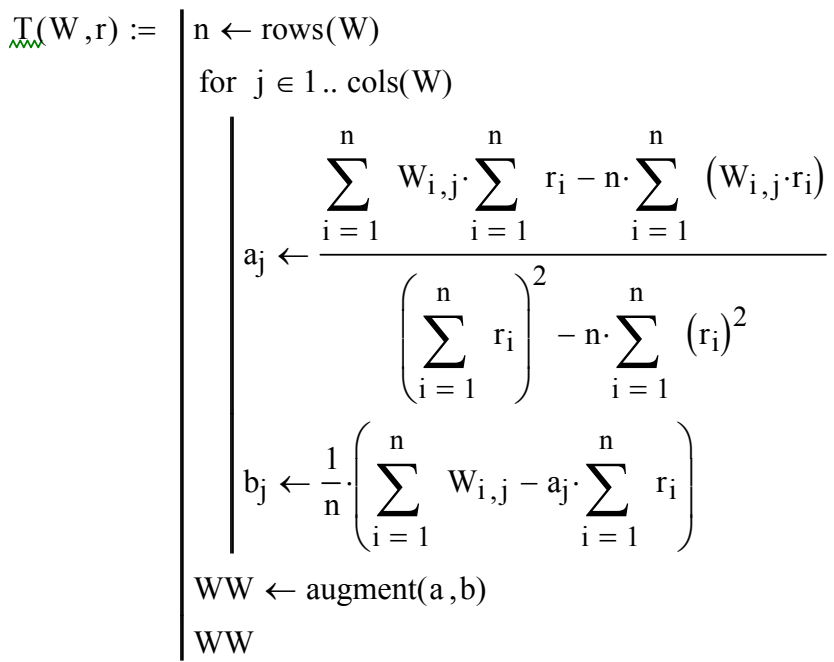


Полученная матрица Т содержит коэффициенты регрессии $(\mathrm{a}, \mathrm{b})$ аппроксимирующих прямых для относительного электропотребления (D) каждого объекта инфраструктуры образовательных учреждений.

$\mathrm{T}(\mathrm{D}, \mathrm{X})^{\mathrm{T}}=$\begin{tabular}{|r|r|r|r|r|r|r|}
\hline & \multicolumn{1}{|c|}{1} & \multicolumn{1}{c|}{2} & \multicolumn{1}{c|}{3} & \multicolumn{1}{c|}{4} & \multicolumn{1}{c|}{5} & \multicolumn{1}{c|}{6} \\
\hline 1 & 0.264 & -0.06 & -0.144 & 0.477 & 0.011 & -0.141 \\
\hline 2 & 3.2 & 4.682 & 2.965 & 2.519 & 4.255 & $\ldots$ \\
\hline
\end{tabular}

Продемонстрируем результаты расчетов на примере объекта под номером $\underset{m}{\mathrm{~N}}:=2$ - средняя школа № 2 (графики - рис. 2).

$$
\left(\mathrm{T}(\mathrm{D}, \mathrm{X})^{\mathrm{T}}\right)^{\langle\mathrm{N}\rangle}=\left(\begin{array}{c}
-0.06 \\
4.682
\end{array}\right)
$$

Коэффициент «а» в уравнении аппроксимирующей прямой тренда равен тангенсу угла наклона данной прямой к оси абсцисс. На рис. 2 для наглядности изображена сопряженная с трендом (черная прямая) горизонтальная красная прямая, которая иллюстрирует угол.

$$
\mathrm{a}:=\left[\left(\mathrm{T}(\mathrm{D}, \mathrm{X})^{\mathrm{T}}\right)^{\langle\mathrm{N}\rangle}\right]_{1}=-0.06 \quad \mathrm{~b}:=\left[\left(\mathrm{T}(\mathrm{D}, \mathrm{X})^{\mathrm{T}}\right)^{\langle\mathrm{N}\rangle}\right]_{2}=4.682 \quad \mathrm{Y}:=\mathrm{a} \cdot \mathrm{X}+\mathrm{b}
$$

Таким образом, для нахождения угла необходимо рассчитать $\operatorname{arctg}(\mathrm{a})$. Это выполняется с помощью подпрограммы Q(M), результат вычислений которой представляет собой вектор $(\Theta \mathrm{rad})$ значений углов наклона прямых(трендов) к оси абсцисс в радианах для каждого объекта.

Листинг 4. Определение индекса жизнеспособности каждого объекта.

$$
\begin{aligned}
& \mathrm{Q}:=\operatorname{atan}(\mathrm{a}) \quad \mathrm{Q}=-0.06 \mathrm{rad} \quad \mathrm{A}:=\mathrm{T}(\mathrm{D}, \mathrm{X})^{\langle 1\rangle} \\
& \mathrm{Qm}(\mathrm{M}):=\mid \begin{array}{l}
\mathrm{n} \leftarrow \text { length }(\mathrm{M}) \\
\text { for } \mathrm{i} \in 1 . . \mathrm{n} \\
\Theta_{\mathrm{i}} \leftarrow \operatorname{atan}\left(\mathrm{M}_{\mathrm{i}}\right) \\
\Theta
\end{array} \\
& \operatorname{erad}:=\mathrm{Q}(\mathrm{A})
\end{aligned}
$$

\begin{tabular}{|c|c|c|c|c|c|c|c|c|}
\hline \multirow[t]{2}{*}{$\Theta^{\mathrm{T}}=$} & & 1 & 2 & 3 & 4 & 5 & 6 & 7 \\
\hline & 1 & 14.77 & -3.42 & -8.21 & 25.5 & 0.61 & -8.05 & $\ldots$ \\
\hline
\end{tabular}

$\operatorname{erad}^{\mathrm{T}}=$\begin{tabular}{|l|l|r|r|r|r|r|}
\hline & 1 & 2 & 3 & 4 & \multicolumn{1}{c|}{5} & 6 \\
\hline 1 & 0.258 & -0.06 & -0.143 & 0.445 & 0.011 & $\ldots$ \\
\hline
\end{tabular}

Пересчитываем радианы в градусы

$$
\Theta:=\frac{\Theta \operatorname{rad} \cdot 180}{\pi}
$$


относит ельное элект ропотребление (\%)

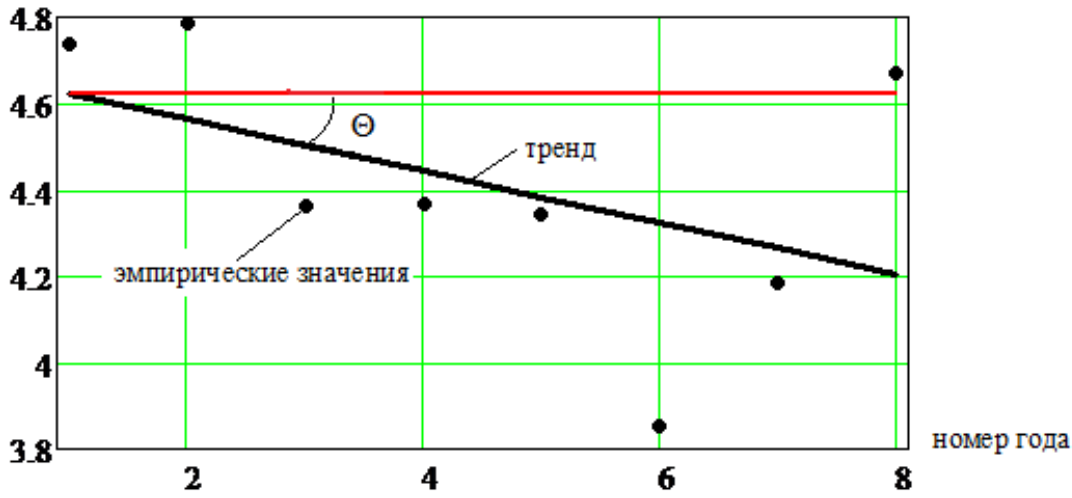

Рис. 2. Макроиндикатор, отражающий динамику электропотребления объекта

и определяем суммарный индекс жизнеспособности всех объектов:

$$
\operatorname{SUM}_{\Theta}:=\sum_{\mathrm{i}=1}^{\mathrm{n}} \Theta_{\mathrm{i}}=-1.807 .
$$

Обратим внимание на то, что чем меньше абсолютное значение индекса жизнеспособности, тем более стабильно и прогнозируемо поведение объекта (в частности, электропотребление) в общей инфраструктуре. Тогда с целью определения первоочередных объектов для углубленного энергетического обследования определяем объекты с наибольшим индексом жизнеспособности, т.е. элементы вектора $\Theta$ с наибольшим значением угла. При этом можно варьировать требуемой долей первоочередных объектов, которая задается параметром Р.

$$
\begin{aligned}
& \mathrm{P}:=6 \\
& \mathrm{Qn}:=\operatorname{submatrix}\left(\operatorname{reverse}(\operatorname{sort}(\Theta)), 1 \text {, round }\left(\frac{\mathrm{n} \cdot \mathrm{P}}{100}\right), 1,1\right)=\left(\begin{array}{c}
25.503 \\
14.773 \\
14.243
\end{array}\right) \\
& \operatorname{Rez}(\mathrm{A}, \mathrm{B}):=\left(\begin{array}{ll}
\mathrm{XY} \leftarrow 0 \\
\text { for } \mathrm{i} \in 1 . . \text { length(B) } \\
\text { for } \mathrm{j} \in 1 . . \text { length }(\mathrm{A}) \\
\mathrm{XY} \leftarrow \mathrm{j} \text { if } \mathrm{B}_{\mathrm{i}}=\mathrm{A}_{\mathrm{j}} \\
\mathrm{XY}
\end{array}\right. \\
& \operatorname{Rez}(\Theta, \mathrm{Q})=\left(\begin{array}{c}
4 \\
1 \\
39
\end{array}\right)
\end{aligned}
$$

\section{Выводы}

Таким образом, по результатам расчетов с применением в качестве макроиндикатора индекса жизнеспособности по электропотреблению выявлены объекты, требующие первоочередного углубленного энергетического обследования. В рассмотренном примере такими являются объекты с номерами в исходной базе данных 4 (СОШ № 13), 1 (СОШ № 1), и 39 ( Детская 
музыкальная школа № 4). Кроме того, объект под номером 4 явно требует особого внимания со стороны системы управления инфраструктурой образовательных учреждений как один из наиболее слабых и стагнирующих.

Предложенный способ позволит создать научно обоснованные предпосылки для проведения целенаправленных углублённых энергетических обследований с последующей реализацией технических и технологических мер по энергосбережению, добиться оптимального управления электропотреблением инфраструктуры бюджетных организаций и учреждений муниципального уровня на системном уровне, а также экономии, полученной за счёт организационных мероприятий, средств, направленных на оплату за потреблённую электроэнергию.

\section{Список литературы}

[1] Кудрин Б.И. Введение в технетику. Томск: Издание ТГУ, 1993. 552 с.

[2] Гнатюк В.И. Закон оптимального построения техноценозов. М.: Изд-во ТГУ. Центр системных исследований, 2005. $384 \mathrm{c}$.

[3] Кудрин Б.И., Жилин Б.В., Лагуткин О.Е., Ошурков М.Г. Ценологическое определение параметров электропотребления многономенклатурных производств. Тула: Приок. кн. изд-во, 1994. $122 \mathrm{c}$.

[4] Гнатюк В.И., Лагуткин О.Е. Ранговый анализ техноценозов. Калининград: БНЦ РАЕН КВИ ФПС РФ, 2000. $86 \mathrm{c.}$

[5] Фуфаев В.В. Ценологическое определение параметров электропотребления, надежности, монтажа и ремонта электрооборудования предприятий региона. М.: Центр системных исследований, 2000. $320 \mathrm{c}$.

[6] Сизганова Е.Ю., Лукьяненко С.Ф. // Энергосбережение и водоподготовка. 2013. №2 (83). C. $72-73$.

[7] Сизганова Е.Ю., Петухов Р.А., Антоненков Д.В., Амузаде А.С. // Вестник ИрГТУ. 2013. № 7. С. $122-127$. 